\title{
Novel Operation Tools with Compression Sensors for Human-Machine Interfaces
}

\author{
Holger Böse, Johannes Ehrlich and Dominik Müller \\ Fraunhofer-Institut für Silicatforschung, Neunerplatz 2, 97082 Würzburg, Germany, \\ E-mail: holger.boese@isc.fraunhofer.de
}

\begin{abstract}
A new class of operation tools as man-machine interfaces for the control of electronic devices is presented. The operation tools exploit the special characteristics of soft and flexible compression sensors. These sensors based on dielectric elastomers have recently been shown to exhibit high sensitivity for compression loads. The basic design of the sensors exhibits two profiled surfaces coated with electrode layers, between which an additional thin elastomer film with the counterelectrode is confined. All components of the sensors are prepared with silicone elastomer whose stiffness can be varied in a wide range. Beside the detection of compression, such sensors can also be used for operation tools in man-machine interfaces, which is demonstrated in three applications. In a first scenario, four sensors were integrated in an automotive steering wheel. By pressing a finger on the sensor, various technical functions can be operated. A second example shows how the sensors can also be used in fabrics to control arbitrary functions of wearable electronic devices. Finally, in another operation tool, a compression sensor is coupled with an electrical switch which also consists of silicone components. With this electrical switch, the desired adjustment of the corresponding technical function controlled by the sensor can be fixed.
\end{abstract}

Key words: Compression sensors, capacitive sensors, flexible sensors, human-machine interfaces, operation tools, compliant substrate, silicone elastomer.

\section{Introduction}

Most human-machine interfaces for the operation of electronic devices or other machines are based on the mechanical input by hand. Examples are keypads, on-off switches, sliders, rotational knobs as well as joysticks and computer mice. This preference refers to the high sensitivity of the human hand, which is capable of applying well-dosed forces using its individual fingers.

In order to quantitatively detect the compression exerted by the finger, suitable sensors are required. Since the fingers are soft and compliant like the whole hand, the sensors recording the applied pressure should exhibit corresponding mechanical properties. Preferably, they are soft, flexible and deformable and adapt to the contours of the skin.

Generally, a large number of compression sensors using different measuring principles are already commercially available today. However, they are commonly hard and mechanically rigid sensors and do not exhibit the desired flexibility and deformability.
The widest distribution of force sensors have strain gauges whose electrical resistance changes significantly already at low deformations. Piezoelectric sensors can yield strong signals even at small deformations as well. However, they react only dynamically, whereas under a static load the electrical signal decreases continuously. Moreover, these sensors are not sufficiently soft and flexible to adapt to the skin and hence less convenient for the use in operation tools.

In contrast, dielectric elastomer sensors (DES) are comparatively very soft, highly flexible and also stretchable. With these features, they are especially convenient for the measurement of strains in one or two dimensions.

Fig. 1, top, shows the basic measuring principle of such strain sensors. A strongly stretchable elastomer film is coated on both sides with also stretchable electrode layers. This configuration represents a deformable electrical plate capacitor. By applying a unidirectional or bidirectional stretch, the area of the elastomer film increases and the thickness decreases. Both effects contribute to an enhancement of the electrical capacitance. As a result, the strain 
of the film is determined by a simple measurement of capacitance [1].
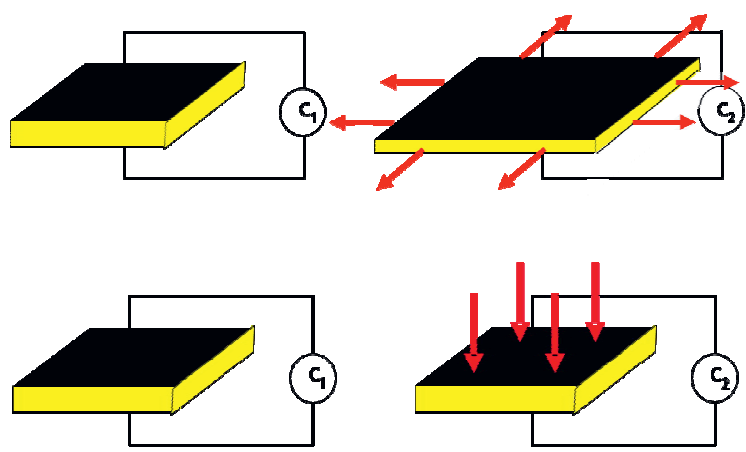

Fig. 1. Schematic representation of the increase of capacitance upon stretching a dielectric elastomer film (top) and no increase of capacitance by compression load on the film (bottom).

Such dielectric elastomer strain sensors are well-suited for the determination of high strains and already commercially available [2]. However, for the measurement of compression loads, they exhibit only very low sensitivity, because on substrates with low compliance, the capacitance changes only very slightly due to the low volume compressibility of the elastomer film (Fig. 1, bottom).

In recent years, different approaches also for flexible capacitive compression sensors were published [3-8]. In some of the sensors, foamed films which under load can be compressed by volume are used as dielectric layers $[4,6,8]$.

Recently, novel compression sensors on the base of dielectric elastomers which exhibit very high sensitivity were developed [9-12]. These capacitive sensors are the fundament of the operating tools described in this paper.

In the following, after a short introduction to the measuring principle of the compression sensors, three possible application scenarios as operation tools are outlined: 1 . the use of sensors on a steering wheel, 2. the integration in fabrics and 3 . the combination with an electrical switch to fix the selected adjustment of the operation tool.

\section{Work Principle of Compression Sensors}

The design of the flexible capacitive compression sensors exhibits a high variability. The preferred version for the operation tools is schematically shown in Fig. 2. The sensor consists of two elastomer films with profiles on their inner surfaces between which a flat elastomer film is confined. Both profiles are coated with electrically connected conducting elastomer layers as the ground electrode of the capacitor. The counter-electrode is located in the intermediate elastomer film and covered on both sides with an insulating dielectric layer. All components of the sensor are made with silicone elastomers. In the electrode layers, carbon black nanoparticles in concentrations above the percolation threshold generate the electrical conductivity.

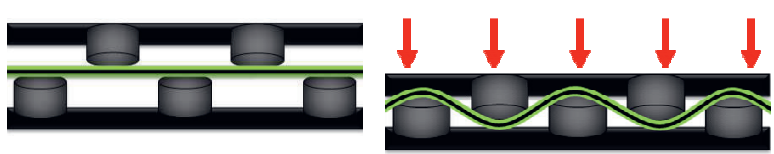

Profile - Dielectric -Electrode

Fig. 2. Scheme of compression sensor with knot profiles.

The profiles on the outer elastomer films are shaped as knots, where the knots of one profile fit into the gaps of the other profile. When the sensor is compressed, the knots of both profiles penetrate into the gaps on the other side, thereby stretching the intermediate elastomer film. With rising compression load, the knots drill into the other profile film and are compressed. All these deformations contribute to a mutual approach of the electrode layers and a corresponding increase of capacitance.

Fig. 3 depicts the result of a continuous increase of the compression load on such a sensor with knot profiles. At a load pressure of $10 \mathrm{~N} / \mathrm{cm}^{2}$, the capacitance of the sensor is more than ten-fold of the initial capacitance. For comparison, a pure dielectric elastomer film sensor exhibits no measurable increase of capacitance under the same conditions (Fig. 3). This analysis emphasizes the very high sensitivity of the new compression sensor.

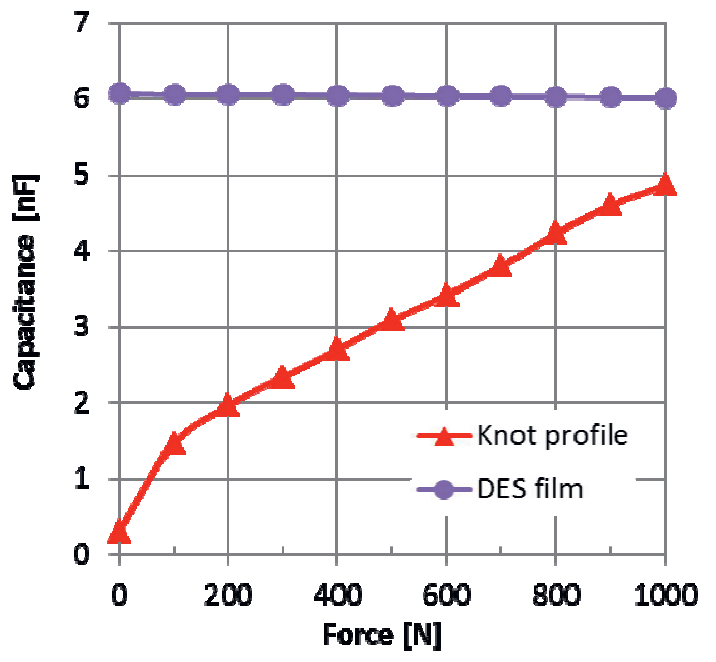

Fig. 3. Dependence of capacitance of a compression sensor with knot profiles on load force compared with pure dielectric elastomer sensor (DES) film (area of both sensors $100 \mathrm{~mm} \times 100 \mathrm{~mm}$ ). 
Fig. 4 reveals three smaller compression sensors with an area of $20 \mathrm{~mm} \times 20 \mathrm{~mm}$, which are used in the operation tools described in the following.

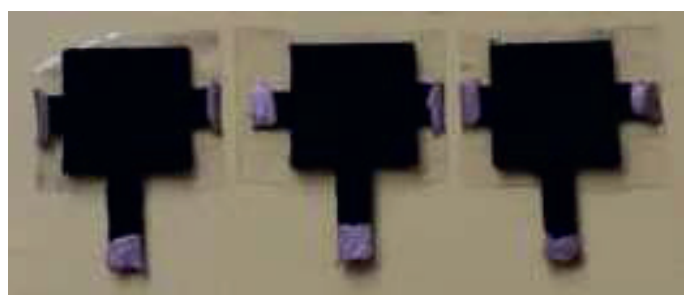

Fig. 4. Compression sensors with knot profiles (area about $20 \mathrm{~mm} \times 20 \mathrm{~mm}$, thickness $3 \mathrm{~mm}$ ).

\section{Applications of the Operation Tools}

Three application scenarios with progressing complexity, in which the dielectric elastomer compression sensors are used as operation tools, will be demonstrated. In a first scenario, the compression sensors are applied in an automotive steering wheel with curved surfaces. The second example shows the use of the soft sensors in a flexible textile structure. Finally, the third application concerns the combination of the sensor with a soft electrical switch.

\section{Steering Wheel}

A possible environment for the use of dielectric elastomer compression sensors as operation tools is the surface of a steering wheel with curved surfaces. To investigate the performance of the compression sensors on this complex surface, an automotive steering wheel was equipped with four compression sensors.
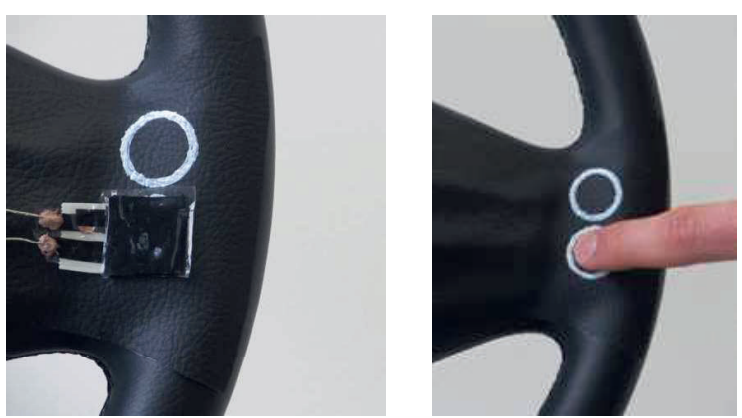

Fig. 5. Steering wheel with compression sensors covered by leather, one of the sensors without cover (left) and finger pressing on the sensor (right).

Fig. 5 shows the steering wheel with the sensors covered by a leather coating. The positions of the sensors are marked by white circles on the leather coating. They are located on the outer part of the wheel and follow its curved shape.

To demonstrate some technical functions, which can be operated with the compression sensors, additional equipment was installed at the steering wheel. Fig. 6 gives an overview on this equipment. One of the compression sensors can regulate the speed of a fan for ventilation. The stronger the finger force on the sensor, the faster the rotation of the fan motor. The neighbored compression sensor sets the temperature of a heater, which is symbolized by continuously changing the color of lights from blue to red upon increasing the finger force. The third compression sensor controls the brightness of a chain of lamps, and, finally, the last sensor regulates the loudness of a speaker.

Obviously, all technical functions can be continuously regulated to an intended adjustment. Fig. 7 shows the symbolized technical functions in more detail. Of course, arbitrary other control functions are also possible.

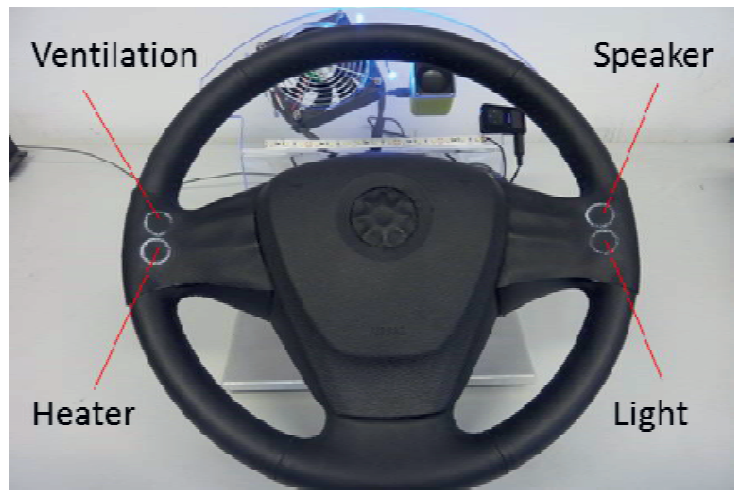

Fig. 6. Demonstrator for a steering wheel with four covered compression sensors as operation tools and assignment to technical functions (ventilation, heating, light and loudspeaker) to be controlled.

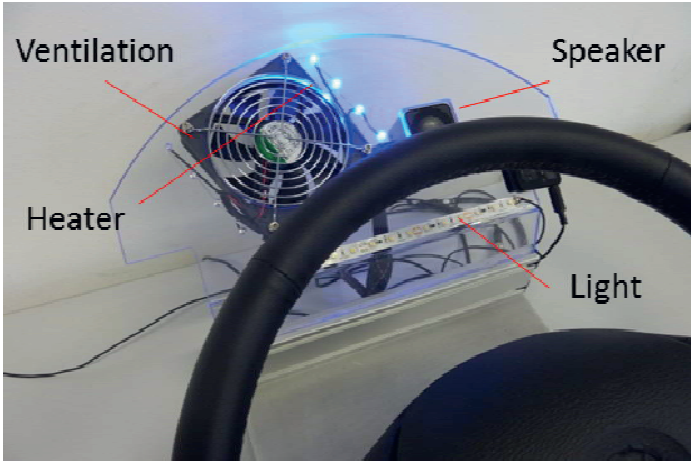

Fig. 7. Details of the demonstrator for a steering wheel with compression sensors as operation tools for technical functions (ventilation, heating, light and loudspeaker) to be controlled. 


\section{Wearables}

A progress of complexity in the environment of the compression sensor for operation tools is demonstrated in the second field of application. The integration of sensors in fabrics requires not only the adaptation of the sensor shape to curved surfaces, but also a high degree of flexibility, softness and compliance. Due to the ongoing strong interest in wearables, operating tools which are suitable of integration in textile structures are needed as well.

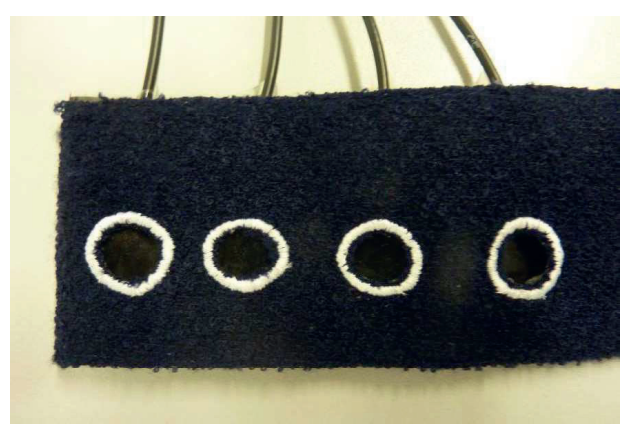

Fig. 8. Textile wristband with four compression sensors as operation tools.

For this purpose, another demonstrator with compression sensors as operation tools was established, which represents a textile wristband to be wrapped around the lower arm. In this demonstrator, four compression sensors are integrated in the wristband depicted in Fig. 8. The white circles on the fabric indicate the position of the circular compression sensors which are accessible for the fingers of the other hand to operate.

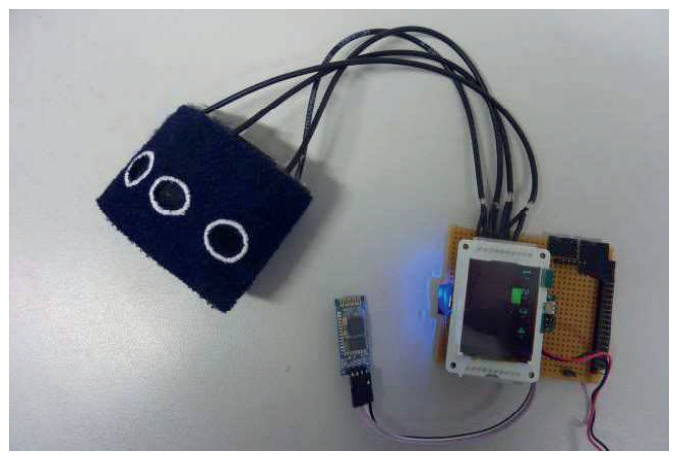

Fig. 9. Demonstrator of textile wristband with four compression sensors as operation tools, electronics and TFT display.

The required electronic equipment for the measurement of the capacitance data, the processing and the visualization was realized with a microcontroller. Fig. 9 shows the demonstrator system with the wristband, the electronics and a small TFT display. In Fig. 10, the wristband wrapped around the lower arm and the electronic board with battery and display are depicted.

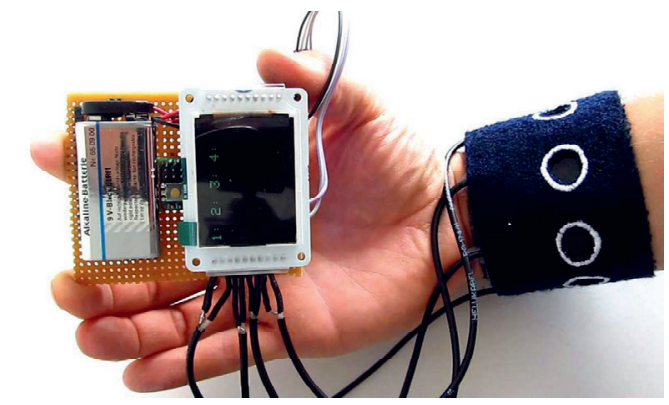

Fig. 10. Demonstrator of textile wristband with four compression sensors as operation tools wrapped around the arm.

In the first version of the demonstrator, the transfer of data from the electronics to the display for the visualization is achieved by direct electrical connections, where the display is mounted directly on the electronic board with the microprocessor. Fig. 11 depicts this configuration in action with two fingers pressing on sensors in the wristband and the corresponding visualization on the TFT display.

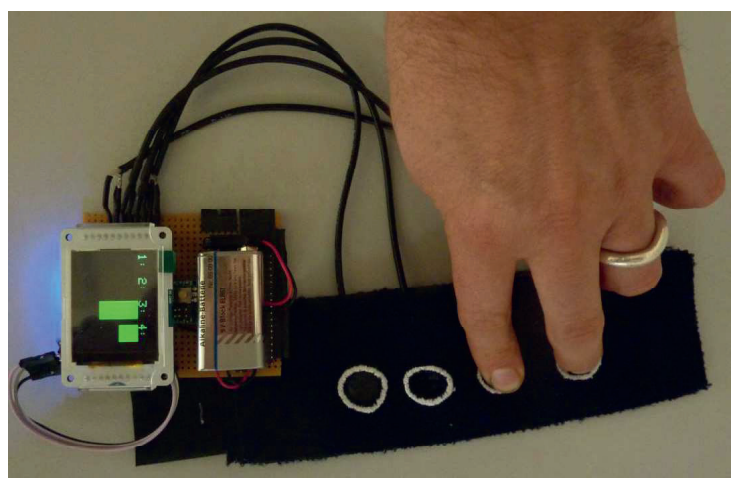

Fig. 11. Demonstrator of textile wristband with four compression sensors as operation tools in action.

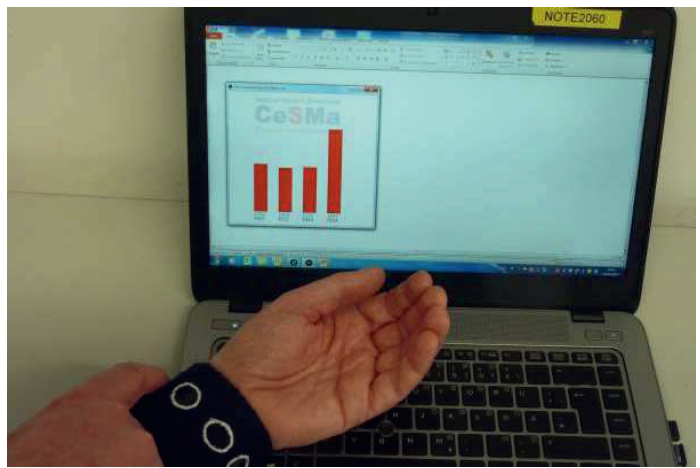

Fig. 12. Transfer of the data of the demonstrator from the textile wristband with four compression sensors as operation tools to a notebook via

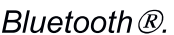


The final version of the demonstrator is equipped with a Bluetooth $\AA$ module connected with the microprocessor, which is capable of wirelessly transferring the capacitance data to a remote device. Fig. 12 reveals this configuration, in which the measured capacitance data of the compression sensors in the wristband are sent to a notebook or a tablet computer where they are visualized on a larger display.

The integration of compression sensors as operation tools in textile structures is a further step to wearables. The operation tools in fabrics can be exploited to control electronic devices. They may be used for the operation of wearable devices such as smartphones or MP3 players. However, such wearable operation tools also offer a benefit for stationary electronic devices such as television and other home appliances, because they can substitute rigid boxes for remote control.

\section{Operation Tool with Electrical Switch}

In the preceding examples for the possible use of compression sensors in operation tools, the target value of the technical function can be adjusted, but when releasing the sensor force, the value is lost. In order to fix this value, an electrical switch which can be triggered without changing the target value is required. This scenario is realized in the next example.

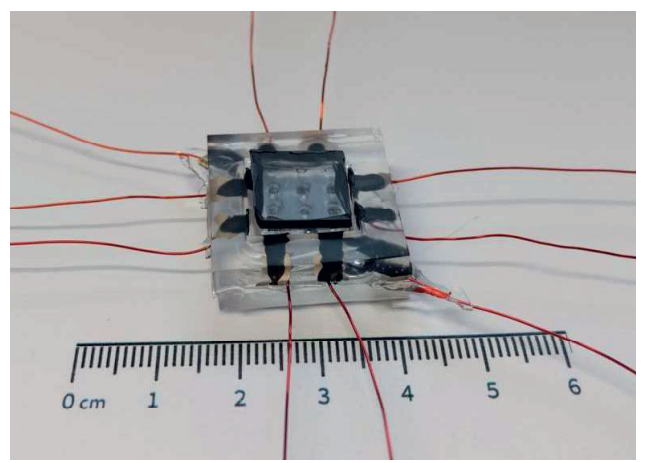

Fig. 13. Basic components of an operation tool with compression sensor and fourfold electrical switch.

Fig. 13 reveals the relevant components of an operation tool with a compression sensor and a fourfold electrical switch. The compression sensor has a design slightly different from that of the former sensors. Here, all electrode layers are located in the intermediate elastomer film between the knot profiles, which leads to an operating range of the sensor shifted to higher forces.

The compression sensor shown in Fig. 13 is surrounded by a soft quadratic frame made with silicone elastomer as well. On all four inner surfaces, the frame is coated with pairs of small electrically conducting areas each, which are connected to the electronic control board to measure the electrical contact between the two conducting areas.

The quadratic compression sensor can also be sheared parallel to its planar surface in all four directions. The sensor is coated with separate electrically conducting areas on the outer side surfaces. When shearing the compression sensor in one of the four directions, the electrical contact between the two conducting areas on the outer frame is closed. This procedure can be performed in all four directions. The four electrical circuits are connected with LEDs in different colors. With this configuration, the brightness of an LED is adjusted by the finger force and the corresponding capacitance of the compression sensor, whereas the color is selected by the direction of the shear motion of the finger.

Fig. 14 depicts the dependence of the capacitance of the compression sensor on the applied force. The graph exhibits a high slope at low forces up to $1.5 \mathrm{~N}$ and a lower slope at high forces above $1.5 \mathrm{~N}$. In the high force region, the brightness of an LED is adjusted and fixed by a shear motion in the corresponding direction. The low force region allows a reset of the fixed brightness of an LED by shearing the compression sensor at a low compression force.

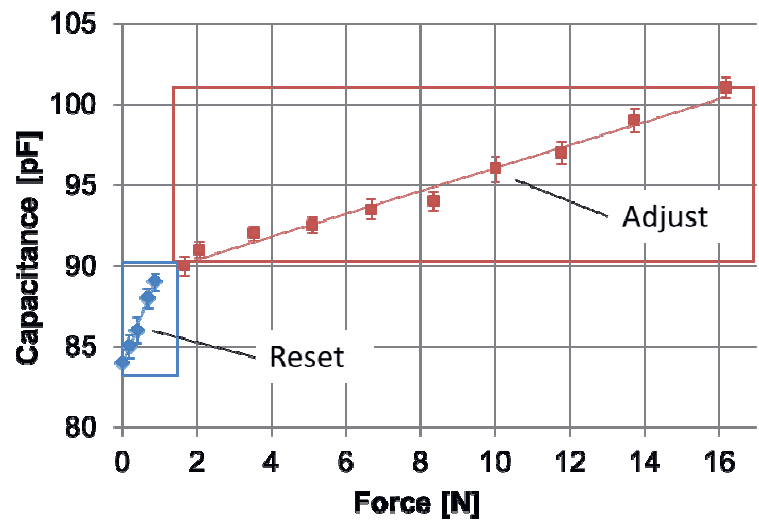

Fig. 14. Dependence of capacitance on applied force on the compression sensor (area $10 \mathrm{~mm} \times 10 \mathrm{~mm}$ ) in the operation tool. At low forces below $1.5 \mathrm{~N}$, the operation tool is reset, at high forces above $1.5 \mathrm{~N}$, the target value is adjusted.

The complete demonstrator of the operation tool with the compression sensor and the fourfold electrical switch is shown in Fig. 15. It allows the independent adjustment of the brightness of four colors. This example demonstrates that even complex procedures can be controlled with the operation tools based on the soft capacitive compression sensors. 


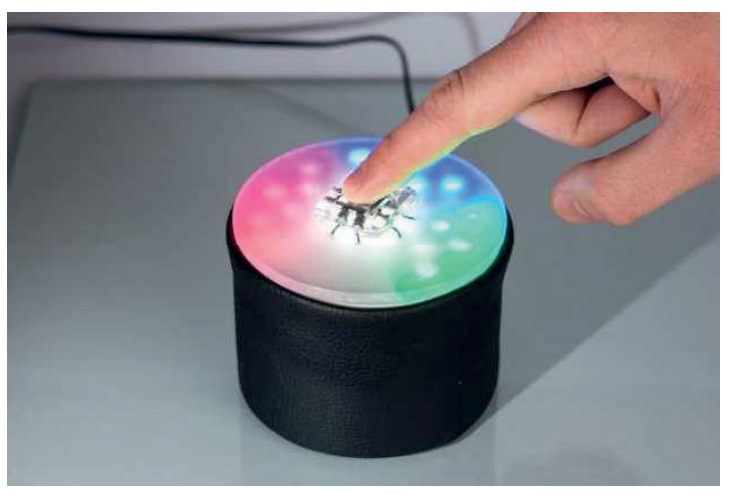

Fig. 15. Operation tool with compression sensor and fourfold electrical switch to adjust and hold the brightness of four LED with different colors.

\section{Conclusions}

In this paper, the work principle and the characteristics of dielectric elastomer compression sensors with knot profiles were described. It could be demonstrated that such capacitive compression sensors exhibit very high sensitivities.

Besides pure sensor applications, the compression sensors have further potential as operation tools for human-machine interfaces. The capacitance signal, which is caused by the compression of a finger on the sensor, can be exploited to control a large number of technical functions. Due to the soft, flexible and compliant behavior of the compression sensors, they can also be used in many unusual environments, where conventional hard and rigid compression sensors fail. This is demonstrated in some examples.

In a first example, soft compression sensors are integrated at different locations of an automotive steering wheel following its curved contours. In a second application scenario, dielectric elastomer compression sensors are integrated in a fabric structure. In this environment, the sensors have to be very soft and flexible. Finally, in a third example, the compression sensor is combined with a fourfold electrical switch. This configuration allows the independent adjustment of various technical functions and the fixing of their target values.

In all application cases of the dielectric elastomer compression sensors, the sensors are connected to a microcontroller, which measures the sensor capacitance in real-time. This combination of soft compression sensors as operation tools and microcontrollers offers interesting perspectives for new humanmachine interfaces.

\section{Acknowledgements}

Financial support for this work from the Bavarian State Ministry for Economy, Infrastructure, Traffic and Technology is gratefully acknowledged.

The authors thank Detlev Uhl and Deniz Ocak for technical support.

\section{References}

[1] F. Carpi, D. De Rossi, R. Kornbluh, R. Pelrine, P.Sommer-Larsen: Dielectric Elastomers as Electromechanical Transducers, Elsevier, 2008

[2] www.stretchsense.com

[3] S.C.B. Mannsfeld et al.: Highly Sensitive Flexible Pressure Sensors with Micro-structured Rubber Dielectric Layers, Nature Materials 9 (2010) 859864

[4] H. Vandeparre, D. Watson, S. P. Lacour: Extremely robust and conformable capacitive pressure sensors based on flexible polyurethane foams and stretchable metallization, Applied Physics Letters 103 (2013) 204103

[5] T. Nakamura, Y. Inoue, D. Kim, N. Matsuhisa, T. Yokota, T. Seketani, T. Someya, M. Sekino: Basic characteristics of implantable flexible pressure sensor for wireless readout using MRI, Conf Proc IEEE Eng Med Biol Soc. (2014) 233841

[6] D. Kwon, T.-I. Lee, M. S. Kim, S. Kim, T.-S. Kim, I. Park: Porous dielectric elastomer based ultrasensitive capacitive pressure sensor and its application to wearable sensing device, Proc. Transducers Conf., Anchorage, Alaska, (2015) 299-302

[7] H. Zhang, M. Y. Wang, J. Li, L. Zhu: A soft compressive sensor using dielectric elastomers. Smart Mater. Struct. 25 (2016) 035045

[8] B.-Y. Lee, J. Kim, H. Kim, C. Kim, S.-D. Lee: Low-cost flexible pressure sensor based on dielectric elastomer film with micro-pores, Sensors and Actuators A 240 (2016) 103-109

[9] H. Böse, E. Fuß: Novel dielectric elastomer sensors for compression load detection, Proc. SPIE Vol. 9056 (2014) 905614 1-13

[10] H. Böse, E. Fuß, P. Lux: Influence of design and material properties on the performance of dielectric elastomer compression sensors, SPIE Vol. 9430 (2015) 943079 1-12

[11] H. Böse, E. Fuß, J. Ehrlich: Capacitive sensor mats for pressure detection, Proc. SENSOR (2015) 55-60, AMA Conferences 19.-21.05.2015, Nuremberg, Germany, DOI 10.5162/sensor2015/A2.2

[12] H. Böse, D. Ocak, J. Ehrlich: Applications of pressure-sensitive dielectric elastomer sensors, Proc. SPIE Vol. 9798 (2016) 97982C 1-13 